\title{
Generalized deviation functions and construction of aggregation functions
}

\author{
Andrea Stupñanová and Peter Smrek \\ Department of Mathematics, FCI, STU in Bratislava \\ Radlinského 11, 81005 Bratislava, Slovakia \\ andrea.stupnanova@stuba.sk, peter.smrek@stuba.sk
}

\begin{abstract}
We generalize the concept of moderate deviation functions $\mathcal{D}:[0,1]^{2} \rightarrow \overline{\mathbb{R}}$ into $(k+1)$ - dimensional moderate deviation function $\mathcal{D}^{(k)}:[0,1]^{k} \times[0,1] \rightarrow \overline{\mathbb{R}}$ and propose a $\mathcal{D}^{(k)}$-based method for constructing idempotent aggregation functions. More, to enable to introduce weights of groups of criteria (coordinates) into our construction method, the concept of normed $(k+1)$-dimensional moderate deviation functions is proposed and exemplified.
\end{abstract}

Keywords: Aggregation function, Deviation function, Moderate deviation function, Penalty function.

\section{Introduction}

The idea of deviation functions can be traced to the deep history. So, for example, standard deviation $D(x, y)=y-x$ of an observation $x$ and its estimate $y$ applied to a sample $\left(x_{1}, \ldots, x_{n}\right)$ yields a global deviation $G\left(x_{1}, \ldots, x_{n}, y\right)=\sum_{i=1}^{n}\left(y-x_{i}\right)$ and its (unique) root $y^{*}, G\left(\mathbf{x}, y^{*}\right)=0$, is just the arithmetic mean $y^{*}$ of our sample $\mathbf{x}$, i.e., $y^{*}=\frac{1}{n} \sum_{i=1}^{n} x_{i}$. Hence the arithmetic mean can be introduced by means of a deviation function. This idea was further formalized by Daróczy [7].

Definition 1.1 Let I be a real interval and let $D$ : $I^{2} \rightarrow \mathbb{R}$ be a function such that

(i) for all $x \in I, D(x, \cdot): I \rightarrow \mathbb{R}$ is continuous and strictly increasing;

(ii) $D(x, x)=0$ for all $x \in I$.

Then the function $D$ is called a deviation function.
It is obvious that for each fixed deviation function $D$ and sample $\mathbf{x}=\left(x_{1}, \ldots, x_{n}\right) \in I^{n}$, the function $G(\mathbf{x}, \cdot): I \rightarrow \mathbb{R}$ given by

$$
G(\mathbf{x}, y)=\sum_{i=1}^{n} D\left(x_{i}, y\right)
$$

is continuous, strictly increasing and attaining value 0 , and thus there is unique solution of the equation

$$
G(\mathbf{x}, y)=0 .
$$

Definition 1.2 Let $D$ be a given deviation function. For each $n \in \mathbb{N}$, the mapping $M_{D}: I^{n} \rightarrow I$ given by $M_{D}(\mathbf{x})=y$, where $y$ is the solution of (1), is called a Daróczy mean.

Clearly, $M_{D}(x, \ldots, x)=x$ for any $x \in I$, and also

$$
\min (\mathbf{x}) \leq M_{D}(\mathbf{x}) \leq \max (\mathbf{x}) .
$$

However, Daróczy means are not monotone, in general, and thus not aggregation functions.

Example 1 Taking the deviation function $D$ : $[-1,1]^{2} \rightarrow \mathbb{R}$ given by $D(x, y)=(x+2)(y-x)$, we can consider global deviation function

$$
\begin{aligned}
G(\mathbf{x}, y) & =\sum_{i=1}^{n}\left(x_{i}+2\right)\left(y-x_{i}\right) \\
& =y \sum_{i=1}^{n}\left(x_{i}+2\right)-\sum_{i=1}^{n} x_{i}\left(x_{i}+2\right) .
\end{aligned}
$$

Then the related Daróczy mean $M_{D}:[-1,1]^{n} \rightarrow$ $[-1,1]$ (as a solution of $(1)$ ) is given by

$$
M_{D}(\mathbf{x})=\frac{\sum_{i=1}^{n} x_{i}\left(x_{i}+2\right)}{\sum_{i=1}^{n}\left(x_{i}+2\right)} .
$$

Then $M_{D}(-1,1)=0.5$ and $M_{D}(-0.9,1)=\frac{201}{410}<0.5$, showing that $M_{D}$ is not an increasing function. 
Several other modification of Daróczy approach where proposed and discussed in the literature, including

- Losonczi means $[11,12]$, where $n$ deviation functions $D_{1}, \ldots, D_{n}$ are considered and (1) is turned into

$$
\sum_{i=1}^{n} D_{i}\left(x_{i}, y\right)=0 .
$$

- Bajraktarevič means [1], where deviation functions $D_{1}, \ldots, D_{n}$ in $(2)$ are considered as

$$
D_{i}(x, y)=w_{i}(x)(s(y)-s(x)),
$$

where $w_{i}: I \rightarrow[0, \infty[$ is a weighting function and $s: I \rightarrow \mathbb{R}$ is continuous and strictly increasing (compare Example 1, where $w(x)=x+2$ and $s(x)=x)$.

- quasi-deviation functions and related quasideviation means proposed by Páles [13].

Observe that several types of mixtures operators (mixture functions) and their generalizations are covered by the above deviation-based approaches, see $[14,15]$. All of these means are not monotone, in general. To avoid this problem and thus to contribute to the aggregation theory we have recently proposed the concept of moderate deviation functions $[8,9]$.

Note first that, for a fixed positive integer $n \in \mathbb{N}$, an aggregation function $A: I^{n} \rightarrow I$ acting on a real interval $I$ is an increasing function satisfying the boundary conditions

$$
\inf \left\{A(\mathbf{x}) \mid \mathbf{x} \in I^{n}\right\}=\inf I
$$

and

$$
\sup \left\{A(\mathbf{x}) \mid \mathbf{x} \in I^{n}\right\}=\sup I .
$$

For more details we recommend [10]. In this contribution we will deal with a fixed real interval $I=[0,1]$, and then boundary conditions (3), (4) for an $n$-ary aggregation function $A:[0,1]^{n} \rightarrow[0,1]$ can be rewritten into

$$
\begin{aligned}
A(0, \ldots, 0) & =0 \\
\text { and } \quad & \\
A(1, \ldots, 1) & =1 .
\end{aligned}
$$

Several important information concerning aggregation functions acting on $I=[0,1]$ can be found in $[2,3,10$, 17].

Definition 1.3 Consider a mapping $\mathcal{D}:[0,1]^{2} \rightarrow \overline{\mathbb{R}}$, (where where $\overline{\mathbb{R}}=\mathbb{R} \cup\{-\infty,+\infty\}$ ) fulfilling (i) for all $x \in[0,1], \mathcal{D}(x, \cdot):[0,1] \rightarrow \overline{\mathbb{R}}$ is increasing (not necessarily strictly);

(ii) for all $y \in[0,1], \mathcal{D}(\cdot, y):[0,1] \rightarrow \overline{\mathbb{R}}$ is decreasing (not necessarily strictly);

(iii) $\mathcal{D}(x, y)=0$ if and only if $x=y \in[0,1]$.

Then $\mathcal{D}$ is called a moderate deviation function, shortly $M D F$. The set of all moderate deviation functions we denoted as $\mathfrak{D}$.

Definition 1.4 Consider moderate deviation function $\mathcal{D} \in \mathfrak{D}$ and $n \in \mathbb{N}$ then the function $\mathcal{G}^{\mathcal{D}}:[0,1]^{n} \times$ $[0,1] \rightarrow \overline{\mathbb{R}}$ given by

$$
\mathcal{G}^{\mathcal{D}}(\mathbf{x}, y)=\sum_{i=1}^{n} \mathcal{D}\left(x_{i}, y\right)
$$

is called global moderate deviation function.

We can introduce modification of equation (1) for moderate deviation functions $\mathcal{D}$,

$$
\mathcal{G}^{\mathcal{D}}(\mathbf{x}, y)=0,
$$

but then, depending on $\mathcal{D} \in \mathfrak{D}$ and $\mathbf{x} \in[0,1]^{n}$, the equation (7) need not have a solution, or it may have more solutions (in the later case, the set of all solutions is an interval). These facts are reflected in the following modification of Definition 1.2.

Definition 1.5 Consider $\mathcal{D} \in \mathfrak{D}$, and $n \in \mathbb{N}$, then the mapping $U_{\mathcal{D}}:[0,1]^{n} \rightarrow[0,1]$ given by

$$
\begin{aligned}
U_{\mathcal{D}}(\mathbf{x})= & \frac{1}{2}\left(\sup \left\{y \in[0,1] \mid \sum_{i=1}^{n} \mathcal{D}\left(x_{i}, y\right)<0\right\}+\right. \\
& \left.+\inf \left\{y \in[0,1] \mid \sum_{i=1}^{n} \mathcal{D}\left(x_{i}, y\right)>0\right\}\right)
\end{aligned}
$$

is called a $\mathcal{D}$-mean.

We recall the standard conventions $\sup \{y \in[0,1] \mid y \in$ $\emptyset\}=0$ and $\inf \{y \in[0,1] \mid y \in \emptyset\}=1$.

Moreover, if $\mathbf{x}=(x, \ldots, x)$, then

$$
\begin{aligned}
& \sum_{i=1}^{n} \mathcal{D}(x, y)<0 \text { if and only if } x<y, \\
& \sum_{i=1}^{n} \mathcal{D}(x, y)>0 \text { if and only if } x>y,
\end{aligned}
$$

and hence $U_{\mathcal{D}}(x, \ldots, x)=x$ for any $x \in[0,1]$.

Theorem 1.1 Let $\mathcal{D} \in \mathfrak{D}$. Then the $\mathcal{D}$-mean $U_{\mathcal{D}}$ : $[0,1]^{n} \rightarrow[0,1]$ is an idempotent symmetric aggregation function. 
Note that there is another closely related concept to deviation functions, namely the penalty functions. Also here they were introduced a studied first based on binary functions $P: I^{2} \rightarrow[0, \infty[$ and then the minimizers of a global penalty $G P(\mathbf{x}, y)=\sum_{i=1}^{n} P\left(x_{i}, y\right)$ were considered to represent the sample $\mathbf{x}$. For more details on penalty-based constructions of aggregation functions we recommend [6, 18], and, in particular [5] and [4]. In these later works, local penalty functions with higher dimensions were proposed and discussed. In particular, this approach has enabled to obtain an arbitrary (idempotent) aggregation function by means of penalty functions. The above idea has inspired us to develop in this contribution more dimensional moderate deviation functions and to apply them for constructing (idempotent) aggregation functions.

Our proposal is introduced, studied and exemplified in the next Section 2. In Section 3, we introduce normed $(k+1)$-dimensional moderate deviation functions and apply them for introduction of weights of groups of criteria (coordinates) into our construction of aggregation functions. Finally, some concluding remarks are added.

\section{2 ( $k+1)$-dimensional deviation functions and construction of aggregation functions}

Definition 2.1 Let $k \in \mathbb{N}$ be fixed. $A(k+1)$ dimensional moderate deviation function is a mapping $\mathcal{D}^{(k)}:[0,1]^{k} \times[0,1] \rightarrow \overline{\mathbb{R}}$ such that

(i) for all $\mathbf{x} \in[0,1]^{k}, \mathcal{D}^{(k)}(\mathbf{x}, \cdot):[0,1] \rightarrow \overline{\mathbb{R}}$ is increasing (not necessarily strictly);

(ii) for all $y \in[0,1], \mathcal{D}^{(k)}(\cdot, y):[0,1]^{k} \rightarrow \overline{\mathbb{R}}$ is decreasing (not necessarily strictly);

(iii) $\mathcal{D}^{(k)}(x, \ldots, x, y)=0$ if and only if $x=y \in[0,1]$.

The set of all $(k+1)$-dimensional moderate deviation functions is denoted as $\mathfrak{D}^{(k)}$.

Example 2 (i) Let $\mathcal{D} \in \mathfrak{D}=\mathfrak{D}^{(1)}$ be a moderate deviation function, then $\mathcal{D}^{(k)}:[0,1]^{k} \times[0,1] \rightarrow \overline{\mathbb{R}}$ given by

$$
\mathcal{D}^{(k)}\left(x_{1}, \ldots, x_{k}, y\right)=\sum_{i=1}^{k} \mathcal{D}\left(x_{i}, y\right)
$$

belongs to $\mathfrak{D}^{(k)}$.

(ii) For any idempotent aggregation function $A$ : $[0,1]^{k} \rightarrow[0,1]$ let $\mathcal{D}^{(k)}:[0,1]^{k} \times[0,1] \rightarrow \overline{\mathbb{R}}$ be given by

$$
\mathcal{D}^{(k)}(\mathbf{x}, y)=y-A(\mathbf{x}) .
$$

Then $\mathcal{D}^{(k)} \in \mathfrak{D}^{(k)}$.

(iii) Let $\mathcal{D}^{(2)}:[0,1]^{2} \times[0,1] \rightarrow \overline{\mathbb{R}}$ be given by

$$
\mathcal{D}^{(2)}\left(x_{1}, x_{2}, y\right)=9 y^{2}-\left(x_{2}+2 x_{1}\right)^{2} \text {. }
$$

Then $\mathcal{D}^{(2)} \in \mathfrak{D}^{(2)}$.

Remark 2.1 Observe that, for any $k \in \mathbb{N}$, the class $\mathfrak{D}^{(k)}$ is closed under positive linear combinations and thus also convex. More, for any $\mathcal{D}^{(k)} \in \mathfrak{D}^{(k)}$ and any permutation $\sigma:\{1, \ldots, k\} \rightarrow\{1, \ldots, k\}$, also $\mathcal{D}^{(k), \sigma}:$ $[0,1]^{k} \times[0,1] \rightarrow \overline{\mathbb{R}}$ given by

$$
\mathcal{D}^{(k), \sigma}\left(x_{1}, \ldots, x_{k}, y\right)=\mathcal{D}^{(k)}\left(x_{\sigma(1)}, \ldots, x_{\sigma(k)}, y\right)
$$

belongs to $\mathfrak{D}^{(k)}$. Also, if $\mathcal{D}^{\left(k_{1}\right)} \in \mathfrak{D}^{\left(k_{1}\right)}$ and $\mathcal{D}^{\left(k_{2}\right)} \in$ $\mathfrak{D}^{\left(k_{2}\right)}$, then $\mathcal{D}^{\left(k_{1}+k_{2}\right)}:[0,1]^{k_{1}+k_{2}} \times[0,1] \rightarrow \overline{\mathbb{R}}$ given by

$$
\begin{array}{r}
\mathcal{D}^{\left(k_{1}+k_{2}\right)}\left(x_{1}, \ldots, x_{k_{1}+k_{2}}, y\right)=\mathcal{D}^{\left(k_{1}\right)}\left(x_{1}, \ldots, x_{k_{1}}, y\right) \\
+\mathcal{D}^{\left(k_{2}\right)}\left(x_{k_{1}+1}, \ldots, x_{k_{1}+k_{2}}, y\right)
\end{array}
$$

belongs to $\mathfrak{D}^{k_{1}+k_{2}}$.

For an injective mapping $\varphi:\{1, \ldots, k\} \rightarrow\{1, \ldots, n\}$, it is obvious that $k \leq n$. We denote $|\varphi|=k$.

Definition 2.2 Fix $n \in \mathbb{N}$, and consider a system $\Phi=\left(\varphi_{1}, \ldots, \varphi_{r}\right)$ of injective functions with co-domain $\{1, \ldots, n\}$, and a related system $\mathbb{D}=\left(\mathcal{D}_{1}, \ldots, \mathcal{D}_{r}\right)$ such that $\mathcal{D}_{i} \in \mathfrak{D}^{\left(/ \varphi_{i} /\right)}, i=1, \ldots, r$. The generalized global moderate deviation function $\mathcal{G}^{\Phi, \mathbb{D}}:[0,1]^{n} \times[0,1] \rightarrow \overline{\mathbb{R}}$ is given by

$$
\mathcal{G}^{\Phi, \mathbb{D}}(\mathbf{x}, y)=\sum_{i=1}^{r} \mathcal{D}_{i}\left(x_{\varphi_{i}(1)}, \ldots, x_{\varphi_{i}\left(/ \varphi_{i} /\right)}, y\right) .
$$

Then a mapping $A_{\Phi, \mathbb{D}}:[0,1]^{n} \rightarrow[0,1]$ given by

$$
\begin{aligned}
A_{\Phi, \mathbb{D}}(\mathbf{x})= & \frac{1}{2}\left(\sup \left\{y \in[0,1] \mid \mathcal{G}^{\Phi, \mathbb{D}}(\mathbf{x}, y)<0\right\}\right. \\
& \left.+\inf \left\{y \in[0,1] \mid \mathcal{G}^{\Phi, \mathbb{D}}(\mathbf{x}, y)>0\right\}\right)
\end{aligned}
$$

is called $a(\Phi, \mathbb{D})$-mean.

The next result validates the fact that the generalized global deviation functions can be applied via (9) as a valid construction method for idempotent aggregation functions.

Theorem 2.1 Under constraints of Definition 2.2, the $(\Phi, \mathbb{D})$-mean $A_{\Phi, \mathbb{D}}:[0,1]^{n} \rightarrow[0,1]$ is an idempotent aggregation function.

Example 3 Let $n=2$. Consider $\Phi=\left(\varphi_{1}, \varphi_{2}\right)$, where $\varphi_{1}:\{1,2\} \rightarrow\{1,2\}$ is given by $\varphi_{1}(1)=2, \varphi_{1}(2)=1$, and $\varphi_{2}:\{1\} \rightarrow\{1,2\}$ is given by $\varphi_{2}(1)=1$. Let 
$\mathcal{D}_{1}=\mathcal{D}^{(2)}$ given in Example 2(iii) and let $\mathcal{D}_{2} \in \mathfrak{D}^{(1)}$ is given by $\mathcal{D}_{2}(x, y)=y-x$. Then the related general global moderate deviation function $\mathcal{G}^{\Phi, \mathbb{D}}$ is given by

$$
\mathcal{G}^{\Phi, \mathbb{D}}\left(x_{1}, x_{2}, y\right)=9 y^{2}-\left(x_{2}+2 x_{1}\right)^{2}+y-x_{1},
$$

and the related idempotent aggregation function $A_{\Phi, \mathbb{D}}$ is given by

$$
A_{\Phi, \mathbb{D}}\left(x_{1}, x_{2}\right)=\frac{1}{18}\left(\sqrt{1+36\left(x_{1}+\left(x_{2}+2 x_{1}\right)^{2}\right)}-1\right) .
$$

\section{Normed $(\mathrm{k}+1)$-dimensional moderate deviation functions}

In our recent contribution [16], we have observed that the introduction of weights for single criteria (coordinates) in $\mathfrak{D}^{(1)}$-based construction of idempotent aggregation functions needs some comparability of extended moderate deviation functions $\mathcal{D}_{1}, \mathcal{D}_{2} \in \mathfrak{D}^{(1)}$. This comparability was proposed to be measured by the values $\mathcal{D}_{1}\left(\frac{1}{4}, \frac{3}{4}\right)$ and $\mathcal{D}_{2}\left(\frac{1}{4}, \frac{3}{4}\right)$, both of them being necessarily positive. If, for $\mathcal{D} \in \mathfrak{D}^{(1)}$ we have the equality $\mathcal{D}\left(\frac{1}{4}, \frac{3}{4}\right)=\frac{1}{2}$, then $\mathcal{D}$ is called a normed moderate deviation function. Obviously, $\frac{\mathcal{D}_{1}}{2 \mathcal{D}_{1}\left(\frac{1}{4}, \frac{3}{4}\right)}$ as well as $\frac{\mathcal{D}_{2}}{2 \mathcal{D}_{2}\left(\frac{1}{4}, \frac{3}{4}\right)}$ are normed moderate deviation function. We propose to extend this concept also for the classes $\mathfrak{D}^{(k)}, k \in \mathbb{N}$.

Definition 3.1 Let $\mathcal{D}^{(k)} \in \mathfrak{D}^{(k)}$ satisfy

$$
\mathcal{D}^{(k)}\left(\frac{1}{4}, \ldots, \frac{1}{4}, \frac{3}{4}\right)=\frac{k}{2} .
$$

Then $\mathcal{D}^{(k)}$ is called a normed $(k+1)$-dimensional moderate deviation function.

The above concept allows to introduce consistently weights into construction method given by (9).

Definition 3.2 Under the constrains and notation of Definition 2.2, let all considered $\mathcal{D}_{i} \in \mathfrak{D}^{\left(/ \varphi_{i}\right) /}$ be normed and let $\left.\mathbf{w}=\left(w_{1}, \ldots, w_{n}\right) \in\right] 0, \infty\left[^{r}\right.$. Then the related weighted generalized global moderate deviation function $\mathcal{G}_{\mathbf{w}}^{\Phi, \mathbb{D}}:[0,1]^{n} \times[0,1] \rightarrow \overline{\mathbb{R}}$ is given by

$$
\mathcal{G}_{\mathbf{w}}^{\Phi, \mathbb{D}}(\mathbf{x}, y)=\sum_{i=1}^{r} w_{i} \mathcal{D}_{i}\left(x_{\varphi_{i}(1)}, \ldots, x_{\varphi_{i}\left(/ \varphi_{i} /\right)}, y\right)
$$

and the related function $A_{\Phi, \mathbb{D}}^{\mathbf{w}}:[0,1]^{n} \rightarrow[0,1]$ is given by

$$
\begin{aligned}
A_{\Phi, \mathbb{D}}^{\mathbf{w}}(\mathbf{x})= & \frac{1}{2}\left(\sup \left\{y \in[0,1] \mid \mathcal{G}_{\mathbf{w}}^{\Phi, \mathbb{D}}(\mathbf{x}, y)<0\right\}\right. \\
& \left.+\inf \left\{y \in[0,1] \mid \mathcal{G}_{\mathbf{w}}^{\Phi, \mathbb{D}}(\mathbf{x}, y)>0\right\}\right) .
\end{aligned}
$$

Theorem 3.1 Under constraints of Definition 3.2, the function $A_{\Phi, \mathbb{D}}^{\mathbf{w}}:[0,1]^{n} \rightarrow[0,1]$ is an idempotent aggregation function.

Note that, for any positive constant $\lambda>0, A_{\Phi, \mathbb{D}}^{\mathbf{w}}=$ $A_{\Phi, \mathbb{D}}^{\lambda \mathbf{w}}$, and thus one can deal with normed weights satisfying $\sum_{i=1}^{r} w_{i}=1$ only. Observe also that we do not consider null weights $w_{i}=0$, as this fact simply means that neither $\varphi_{i}$ nor $\mathcal{D}_{i}$ are considered in construction (10).

Example 4 Continuing in Example 3, let $w_{1}=\lambda \in$ ]0, $1\left[\right.$ and $w_{2}=1-\lambda$. Then $\mathcal{D}_{2}\left(\frac{1}{4}, \frac{3}{4}\right)=\frac{3}{4}-\frac{1}{4}=0.5$, i.e., $\mathcal{D}_{2}$ is normed. On the other side,

$$
\mathcal{D}_{1}\left(\frac{1}{4}, \frac{1}{4}, \frac{3}{4}\right)=\frac{81}{16}-\frac{9}{16}=\frac{9}{2} \neq \frac{2}{2}=1,
$$

i.e., $\mathcal{D}_{1}$ is not normed. However,

$$
\overline{\mathcal{D}}_{1}=\frac{\mathcal{D}_{1}}{\mathcal{D}_{1}\left(\frac{1}{4}, \frac{1}{4}, \frac{3}{4}\right)}=\frac{2}{9} \mathcal{D}_{1}
$$

is normed. Denote $\overline{\mathbb{D}}=\left(\overline{\mathcal{D}}_{1}, \mathcal{D}_{2}\right)$. Then the idempotent aggregation function $A_{\Phi, \overline{\mathbb{D}}}^{\mathbf{w}}:[0,1]^{2} \rightarrow[0,1]$ is given by

$A_{\Phi, \overline{\mathbb{D}}}^{\mathbf{w}}\left(x_{1}, x_{2}\right)=\frac{1}{4 \lambda}\left(\sqrt{g_{\lambda}\left(x_{1}, x_{2}\right)}-1+\lambda\right)$, where

$g_{\lambda}\left(x_{1}, x_{2}\right)=(1-\lambda)^{2}+\frac{16}{9} \lambda^{2}\left(x_{2}+2 x_{1}\right)^{2}+8 \lambda(1-\lambda) x_{1}$.

Observe that if $\lambda \rightarrow 1^{-}$then $A_{\Phi, \overline{\mathbb{D}}}^{\mathbf{w}}\left(x_{1}, x_{2}\right) \rightarrow$ $\frac{x_{2}+2 x_{1}}{3}$ (i.e., we are close to the weighted arithmetic mean which is the unique root of the equation $D_{1}\left(x_{2}, x_{1}, y\right)=0$, when $x_{1}, x_{2}$ are fixed).

Similarly, if $\lambda \rightarrow 0^{+}$then $A_{\Phi, \overline{\mathbb{D}}}^{\mathrm{w}}\left(x_{1}, x_{2}\right) \rightarrow x_{1}$ (i.e., we are close to the first projection which is the unique root of the equation $\mathcal{D}_{2}\left(x_{1}, y\right)=0$, when $x_{1}$ is fixed).

\section{Concluding remarks}

We have extended the concept of (normed) moderate deviation functions for higher dimensions. Based on these deviation functions, a new type of construction for idempotent aggregation functions was proposed and exemplified. Note that our approach allows to predetermine attitudes of some groups of arguments, as well as to consider weights of such groups. We expect several interesting applications in the decision making domains, which will be the topic of our further study.

\section{Acknowledgement}

This work was supported by the Slovak Research and Development Agency under the contract no. APVV17-0066 and grant VEGA 1/0682/16. 


\section{References}

[1] M. Bajraktarevič, Über die vergleichbarkeit der mit gewichtsfunktionen gebildeten mittelwerte, Stud. Math. Hungar. 4 (1969) 3-8.

[2] G. Beliakov, H. Bustince Sola, T. Calvo Sánchez, A Practical Guide to Averaging Functions, Springer, 2016.

[3] G. Beliakov, A. Pradera, T. Calvo, A Guide for Practitioners, Springer, 2007.

[4] H. Bustince, G. Beliakov, G. Dimuro, B. Bedregal, R. Mesiar, On the definition of penalty functions in data aggregations, Fuzzy Sets and Systems 323 (2017) 1-18.

[5] T. Calvo, G. Beliakov, Aggregation functions based on penalties, Fuzzy Sets and Systems 161 (10) (2010) 1420-1436.

[6] T. Calvo, R. Mesiar, R. Yager, Quantitative weights and aggregation, IEEE Transactions on Fuzzy Systems 12 (1) (2004) 62-69.

[7] Z. Daróczy, Über eine klasse von mittelwerten, Publ. Math. Debrecen 19 (1972) 211-217.

[8] M. Decký, R. Mesiar, A. Stupňanová, Aggregation functions based on deviations, in: J. M. et al (Ed.), CCIS, Proc. of IPMU 2018, Vol. 853, Springer, 2018, pp. 151-159.

[9] M. Decký, R. Mesiar, A. Stupňanová, Deviationbased aggregation functions, Fuzzy Sets and Systems 332 (2018) 29-36.

[10] M. Grabisch, J. Marichal, R. Mesiar, E. Pap, Aggregation Functions, Cambridge University Press, Cambridge, 2009.

[11] L. Losonczi, General inequalities of nonsymmetric means, Aequationes Mathematicae 9 (1973) 221-235.

[12] L. Losonczi, Hölder-type inequalities, GI 3 (1981) 91-105.

[13] Z. Páles, On homogeneous quasideviation means, Aequationes Mathematicae 36 (2-3) (1988) 132152.

[14] R. Ribeiro, R. M. Pereira, Generalized mixture operators using weighting functions: a comparative study with wa and owa, European Journal of Operational Research 145 (2003) 329-342.

[15] J. Špirková, Induced weighted operators based on dissimilarity functions, Information Sciences 294 (2015) 530-539.
[16] A. Stupňanová, Decisions based on deviation functions, in: Proc. 6th International Conference on Control, Decision and Information Technologies (CODIT'19), Paris, France, 2019, submitted.

[17] V. Torra, Y. Narukawa, Modeling Decisions: Information Fusion and Aggregation Operators, Springer, Berlin, 2007.

[18] R. Yager, Toward a general theory of information aggregation, Information Sciences 68 (3) (1993) 191-206. 